\title{
Horizontal transfer of microRNAs: molecular mechanisms and clinical applications
}

\author{
Xi Chen, Hongwei Liang, Junfeng Zhang ${ }^{\bowtie}$, Ke Zen ${ }^{\bowtie}$, Chen-Yu Zhang ${ }^{凶}$ \\ Jiangsu Engineering Research Center for microRNA Biology and Biotechnology, State Key Laboratory of Pharmaceutical \\ Biotechnology, School of Life Sciences, Nanjing University, Nanjing 210093, China \\ $\bowtie$ Correspondence: cyzhang@nju.edu.cn (C. Zhang), kzen@nju.edu.cn (K. Zen), jfzhang@nju.edu.cn (J. Zhang) \\ Received December 9, 2011 Accepted December 30, 2011
}

\section{ABSTRACT}

A new class of RNA regulatory genes known as microRNAs (miRNAs) has been found to introduce a whole new layer of gene regulation in eukaryotes. The intensive studies of the past several years have demonstrated that miRNAs are not only found intracellularly, but are also detectable outside cells, including in various body fluids (e.g. serum, plasma, saliva, urine and milk). This phenomenon raises questions about the biological function of such extracellular miRNAs. Substantial amounts of extracellular miRNAs are enclosed in small membranous vesicles (e.g. exosomes, shedding vesicles and apoptotic bodies) or packaged with RNA-binding proteins (e.g. high-density lipoprotein, Argonaute 2 and nucleophosmin 1). These miRNAs may function as secreted signaling molecules to influence the recipient cell phenotypes. Furthermore, secreted extracellular miRNAs may reflect molecular changes in the cells from which they are derived and can therefore potentially serve as diagnostic indicators of disease. Several studies also point to the potential application of siRNA/ miRNA delivery as a new therapeutic strategy for treating diseases. In this review, we summarize what is known about the mechanism of miRNA secretion. In addition, we describe the pathophysiological roles of secreted miRNAs and their clinical potential as diagnostic biomarkers and therapeutic drugs. We believe that miRNA transfer between cells will have a significant impact on biological research in the coming years.

KEYWORDS microRNA, extracellular microRNA, microRNA secretion, horizontal transfer, microvesicle, exosome, apoptotic body, high-density lipoprotein, Argonaute 2, nucleophosmin 1, diagnosis, therapy

\section{INTRODUCTION}

Virtually all organisms disseminate genetic material by vertical gene transfer in which genetic information is transmitted from parents to offspring. In contrast, the process of horizontal gene transfer, also known as lateral gene transfer, refers to the movement of genetic information across normal mating barriers between more or less distantly related organisms (Keeling and Palmer, 2008; Holmgren, 2010; Dunning Hotopp, 2011). Horizontal gene transfer is an effective mechanism for the exchange of genetic information in bacteria, allowing bacterial diversification and facilitating adaptation to new environments. With the development of higher eukaryotes, the intercellular exchange of genetic material is restricted (Keeling and Palmer, 2008; Holmgren, 2010; Dunning Hotopp, 2011). However, recent reports have described the intriguing idea that the intercellular exchange of genetic material may still occur in multicellular organisms through several distinct pathways (Holmgren et al., 1999; Bergsmedh et al., 2001; Ratajczak et al., 2006; Davis, 2007; Deregibus et al., 2007; Valadi et al., 2007; Rechavi et al., 2009; Ahmed and Xiang, 2011). As such, comprehensive reviews have discussed the potential roles of nanotubes, exosomes, apoptotic bodies and nucleic acid-binding peptides in the intercellular transfer of genetic information (Belting and Wittrup, 2008).

The phenomenon of microRNA (miRNA) secretion and its role in intercellular crosstalk has recently gained increasing attention. Intensive studies have provided evidence that the exosomes, shedding vesicles, apoptotic bodies and proteinmiRNA complexes that are released from many cell types can transfer miRNAs to neighboring or distant cells, playing a previously unrecognized role in modulating cell functions (Valadi et al., 2007; Zernecke et al., 2009; Kosaka et al., 2010a; Wang et al., 2010; Zhang et al., 2010; Arroyo et al., 
2011; Turchinovich et al., 2011; Vickers et al., 2011). In this review, we summarize the recent literature on the mechanism of miRNA secretion and discuss its potential roles in eukaryotes. We also highlight the potential use of secreted miRNAs as diagnostic biomarkers and therapeutic drugs in clinical practice.

\section{INTERCELLULAR TRANSFER OF DNA, mRNA AND PROTEIN}

Several reports support the existence of horizontal DNA transfer between cells (Holmgren et al., 1999; Bergsmedh et al., 2001). For example, apoptotic bodies released from tumor cells can deliver oncogenic DNA and transform non-malignant surrounding cells (Bergsmedh et al., 2001). One of the first studies to provide evidence for the horizontal transfer of mRNA between cells was performed by Ratajczak et al., who found that microvesicles derived from embryonic stem cells could induce phenotypic changes in hematopoietic progenitor cells not only by binding to surface-expressed ligands but also by delivery of specific mRNAs (Ratajczak et al., 2006). At least two other cases for mRNA transfer have been observed. Endothelial progenitor cell-derived microvesicles can activate an angiogenic program in endothelial cells by the horizontal transfer of mRNAs (Deregibus et al., 2007). In addition, exosomes from mouse mast cells can donate their mRNAs to human mast cells, leading to expression of new mouse proteins in human mast cells and suggesting that exosomes can transfer biological information across species (Valadi et al., 2007). Finally, intercellular protein exchange has long been the subject of intensive investigations (Davis, 2007; Rechavi et al., 2009; Ahmed and Xiang, 2011). Intercellular protein transfer can occur via the internalization pathway, the dissociation-associated pathway, exosome uptake and membrane nanotube formation (Davis, 2007; Rechavi et al., 2009; Ahmed and Xiang, 2011).

\section{MECHANISMS OF INTERCELLULAR TRANSFER OF miRNAs}

\section{Intracellular miRNA}

miRNAs are naturally occurring, small, non-coding RNAs of approximately 22 nucleotides in length (Ambros, 2004; Bartel, 2004; He and Hannon, 2004). Mature miRNAs are generated from primary miRNA transcripts (pri-miRNAs), which are often several thousand nucleotides long, by two sequential cleavages (Lee et al., 2004). The first step of miRNA cleavage is catalyzed in the nucleus by the RNase III enzyme Drosha to generate a 70 to 100 nucleotides long hairpin miRNA precursor (pre-miRNA) with a 2 nucleotide overhang at the 3 '-end (Lee et al., 2003). Following cleavage, pre-miRNA is transported from the nucleus to the cytoplasm by an Exportin5-dependent mechanism (Lund et al., 2004). Once in the cytoplasm, pre-miRNA is cleaved by the RNase III enzyme
Dicer into $\sim 22$ nucleotides long miRNA duplex (Hutvágner et al., 2001). Next, one strand is selected for incorporation into a ribonucleoprotein complex called the RNA-induced silencing complex (RISC) and will function as a mature miRNA. The key components of RISC are proteins of the Argonaute family, and Argonaute 2 (AGO2) is thought to play a critical role in miRNA-mediated mRNA silencing (Khvorova et al., 2003; Schwarz et al., 2003). Mature miRNA acts as a guide to target RISC complex to a complementary mRNA sequence which is inactivated either by cleavage or translational interference, depending on the degree of complementarity between the miRNA and its target (Ambros, 2004; Bartel, 2004; He and Hannon, 2004). In animals, single-stranded miRNA binds through partial sequence homology to the $3^{\prime}$ untranslated region ( $3^{\prime}$ UTR) of the target mRNAs and causes either a translational block or, less frequently, mRNA degradation (Ambros, 2004; Bartel, 2004; He and Hannon, 2004).

miRNAs comprise one of the most abundant classes of gene regulatory molecules in diverse organisms and are estimated to regulate the expression of greater than $60 \%$ of all protein-coding genes. As such, miRNAs play vital roles in all biological processes, including proliferation, differentiation, cell growth, cell death, stress resistance and fat metabolism (Ambros, 2004; Bartel, 2004; He and Hannon, 2004). Deviations from the normal expression pattern of miRNAs likely play a role in diseases such as cancer and heart disease (Calin and Croce, 2006; Esquela-Kerscher and Slack, 2006; van Rooij and Olson, 2007).

\section{Extracellular miRNA}

While the majority of miRNAs are found intracellularly, a number of miRNAs have recently been detected outside of cells, including in various body fluids (e.g., serum, plasma, saliva, urine and milk) (Chen et al., 2008; Lawrie et al., 2008; Mitchell et al., 2008; Park et al., 2009; Chen et al., 2010; Hanke et al., 2010; Kosaka et al., 2010b). Furthermore, alterations in the level and composition of these extracellularcirculating miRNAs are tightly correlated with various health problems, including cancers (Chen et al., 2008; Lawrie et al., 2008: Mitchell et al., 2008; Park et al., 2009; Hanke et al., 2010), diabetes (Chen et al., 2008) and tissue injury (Ji et al., 2009; Laterza et al., 2009; Wang et al., 2009). These results firmly establish the quantification of circulating miRNAs as an extremely promising biomarker to assess and monitor the body's pathophysiological status.

Extracellular miRNAs circulate in the body fluids with a high concentration and sufficient integrity despite high extracellular ribonuclease (RNase) activity, indicating that extracellular miRNAs are likely packaged in some manner to shield them from digestion. Indeed, naked miRNAs added to plasma are immediately degraded, whereas circulating miRNAs are stable for hours under the same conditions (Mitchell et al., 2008). Further studies have also shown that circulating miRNAs are protected from and resistant to harsh conditions 
such as extreme temperatures, extreme $\mathrm{pHs}$ or freeze-thaw cycles (Chen et al., 2008). This phenomenon raises intriguing questions regarding the mechanism of circulating miRNA stability. Furthermore, the origin of such extracellular miRNAs also requires exploration. At present, there are at least three possibilities for the remarkable stability of circulating miRNAs and their sources: (1) they are passively leaked from broken cells during tumorigenesis or tissue injury (Chen et al., 2008; Mitchell et al., 2008); (2) they are packaged in small membranous vesicles, including exosomes, shedding vesicles and apoptotic bodies, which accounts for the release of miRNAs into circulation and offers protection against RNase activity (Valadi et al., 2007; Zernecke et al., 2009; Kosaka et al., 2010a; Zhang et al., 2010); (3) they are protected by the formation of a protein-miRNA complex. Some studies have demonstrated that many extracellular miRNAs are detectable in conjunction with proteins or lipoproteins, but devoid of membrane vesicles, suggesting the existence of non-vesicleenclosed, RNA-binding protein-associated miRNAs in extracellular fluids, including those bound to AGO2, nucleophosmin 1 (NPM1), and high-density lipoprotein (HDL) (Wang et al., 2010; Arroyo et al., 2011; Turchinovich et al., 2011; Vickers et al., 2011).

Recent studies indicate that miRNAs released from donor cells that are either enclosed in vesicles (exosomes, shedding vesicles and apoptotic bodies) or associated with lipoproteins (HDL) are active and can function as secreted molecules to influence the recipient's cell phenotype (Zernecke et al., 2009; Kosaka et al., 2010a; Zhang et al., 2010; Vickers et al., 2011). The difference between vesicleenclosed and lipoprotein-associated miRNAs is largely unknown. Their different secretion mechanisms suggest that they may originate from different cell types and, therefore, have different fates and functions. For example, miRNAs detected only in the lipoprotein-associated fractions may be generated by cells with lipoprotein transport pathways. By contrast, miRNAs that are predominantly packaged in vesicles may originate from cell types known to generate abundant vesicles. However, miRNAs associated with other types of RNA-binding proteins (e.g., AGO2 and NPM1) may also be actively released from donor cells and taken up by recipient cells, although the direct evidence remains elusive. A model of the miRNA secretion via three different pathways is summarized in Fig. 1.

\section{Microvesicle-mediated miRNA transfer}

Based on size, intracellular origin and release mechanism, there are two distinct types of microvesicles that can be secreted by cells: exosomes and shedding vesicles (Théry et al., 2002; Cocucci et al., 2009; Simons and Raposo, 2009). Exosomes are small vesicles of approximately 30 to $100 \mathrm{~nm}$ in size that are formed from endosomes (Théry et al., 2002; Simons and Raposo, 2009). Endosomes, which arise from an inward budding of the plasma membrane into the cytoplasm, invaginate to form multivesicular bodies (MVBs) (Théry et al., 2002; Simons and Raposo, 2009). Exosomes are stored as intraluminal vesicles in MVBs (Théry et al., 2002; Simons and Raposo, 2009). The exocytic MVB then fuses with the plasma membrane, releasing its cargo of exosomes into the extracellular space (Théry et al., 2002; Simons and Raposo, 2009). By contrast, shedding vesicles are plasma membranederived vesicles that are released into the extracellular space by outward budding of the plasma membrane (Cocucci et al., 2009). Shedding vesicles are much larger than exosomes and are heterogeneous in size, ranging from $100 \mathrm{~nm}$ to $1 \mu \mathrm{m}$ (Cocucci et al., 2009). Their relatively large size and the presence of specific molecules are often used to distinguish shedding vesicles from exosomes. The microvesicle population that has been observed in vascular circulation is a mixture of both shedding vesicles and exosomes and can be released by many different cell types (Théry et al., 2002; Cocucci et al., 2009; Simons and Raposo, 2009). Although they were initially considered "cell debris," microvesicles have now emerged as important regulators in cellular crosstalk, influencing diverse biological functions (Théry et al., 2002; Cocucci et al., 2009; Simons and Raposo, 2009).

Microvesicles can stimulate target cells either via transfer of genetic materials or by binding to specific surface receptors (Théry et al., 2002; Cocucci et al., 2009; Simons and Raposo, 2009). Microvesicle-mediated transfer of bioactive proteins, lipids and mRNAs between cells has also been well documented (Théry et al., 2002; Cocucci et al., 2009; Simons and Raposo, 2009). Recent studies have demonstrated that functional miRNAs are also horizontally transferred via this mechanism. The presence of a miRNA pool has been reported in exosomes and shedding vesicles derived from a variety of sources, including mast cells (Valadi et al., 2007), blood (Hunter et al., 2008), stem cells (Yuan et al., 2009; Collino et al., 2010), adipocytes (Ogawa et al., 2010; Müller et al., 2011), syncytiotrophoblasts (Luo et al., 2009) and milk (Hata et al., 2010). Valadi et al. pioneered the description of the so-called "exosomal shuttle miRNA," showing that exosomes isolated from a mouse mast cell line $\mathrm{MC} / 9$, a human mast cell line HMC-1 and from primary bone marrow-derived mast cells contain substantial miRNA populations (Valadi et al., 2007). In a study by Hunter et al., they found significant differences in the expression levels of miRNAs in plasma microvesicles compared with that in platelets and peripheral blood mononuclear cells of healthy donors (Hunter et al., 2008). Based on this result, they predicted that miRNAs in plasma microvesicles are important factors in the regulation of hematopoiesis (Hunter et al., 2008). Yuan et al. demonstrated that microvesicles derived from embryonic stem cells contained abundant miRNAs that can be transferred to mouse embryonic fibroblasts in vitro (Yuan et al., 2009). In another study, Collino et al. demonstrated that microvesicles released from human bone marrow-derived mesenchymal stem cells and liver resident stem cells contained miRNAs and that 


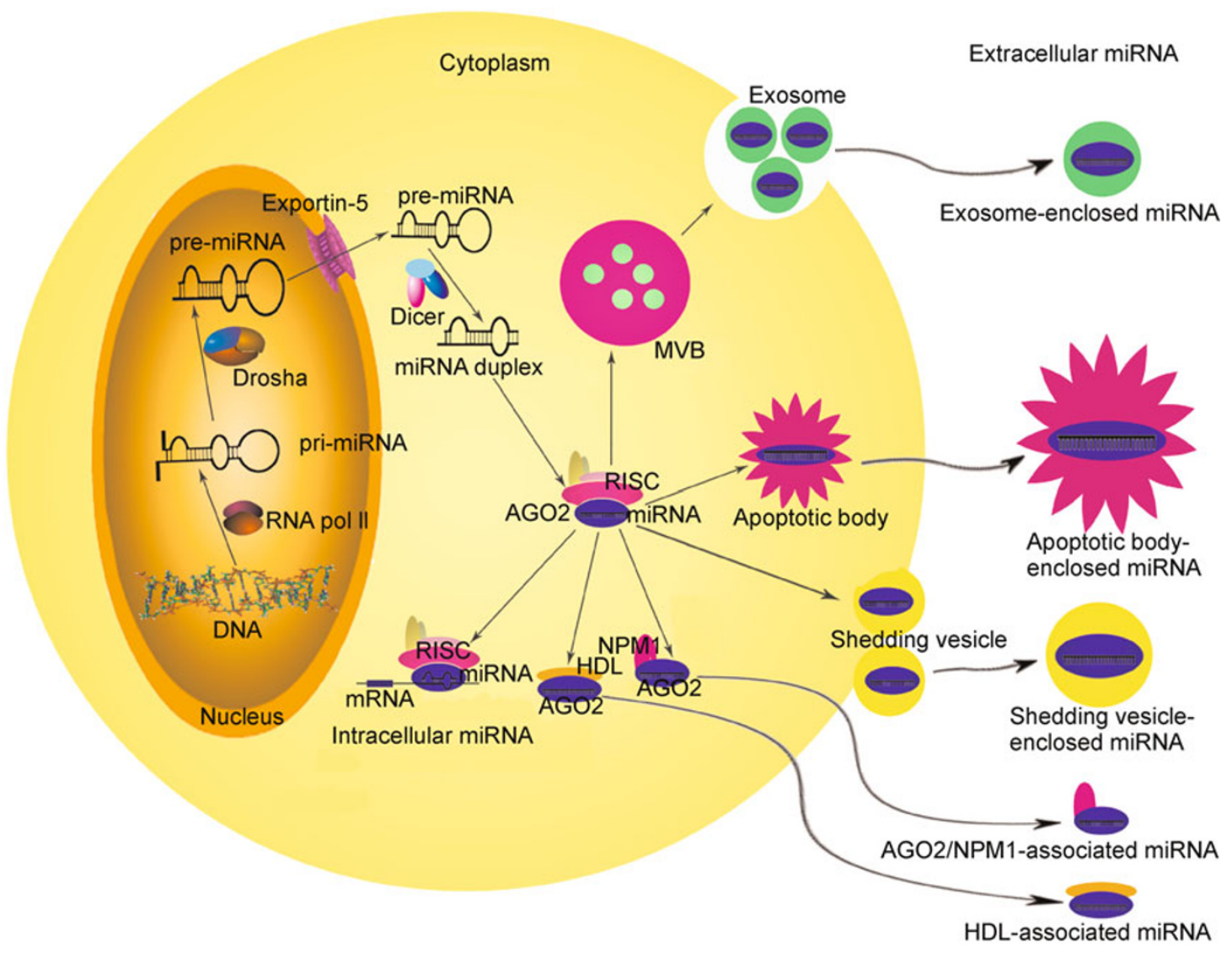

Figure 1. A model of the secretion of miRNAs. After being processed to the mature form, some miRNAs can bind to complementary sequences on target mRNAs to repress translation or trigger mRNA cleavage. Other miRNAs are packaged and transported to the extracellular environment via three different pathways: (1) they are enclosed within membranous vesicles, including exosomes, shedding vesicles and apoptotic bodies; (2) they are associated with lipoproteins, such as HDL; (3) they are associated with RNA-binding proteins, such as AGO2 and NPM1. AGO2, Argonaute 2; NPM1, nucleophosmin1.

microvesicle-enriched miRNAs were transferred to recipient cells after microvesicle incorporation (Collino et al., 2010). These findings raise the intriguing possibility that stem cells can alter the expression of genes in neighboring cells by transferring microvesicle-enclosed miRNAs. Ogawa et al. found that microvesicles secreted by cultured 3T3-L1 adipocytes harbored nearly 140 miRNAs, the majority of which were adipocyte-specific and abundantly expressed in the donor adipocytes (Ogawa et al., 2010). In another study, Müller et al. detected a number of miRNAs in microvesicles released from primary and differentiated rat adipocytes, as well as in microvesicles from rat serum (Müller et al., 2011). However, the biological significance of miRNAs derived from adipocyte microvesicles remains uncertain at this moment. In a different system, Luo et al. hypothesized that syncytiotrophoblasts secrete miRNAs into maternal circulation via exosomes (Luo et al., 2009). In an attempt to prove this hypothesis, they isolated exosomes from the villous trophoblast model cell line, BeWo. The placenta-specific miRNA
miR-517a was detected in the exosome-enriched fraction (Luo et al., 2009). This finding suggests that placenta-specific miRNAs are released extracellularly via exosomes from chorionic villous trophoblasts into maternal circulation, where they could target maternal tissues (e.g. maternal endothelium) during pregnancy. A recent study by Hata et al. showed that several miRNAs thought to be expressed in either mammary glands or immune cells were present in milk-derived microvesicles at considerable levels (Hata et al., 2010). When milk was acidified to mimic its response to gastrointestinal tract conditions, the abundance and quality of microvesicle-derived miRNA were largely unaffected (Hata et al., 2010), suggesting that milk miRNAs could reach innate and acquired immune cells in gut-associated lymphoid tissues of sucking calves. Further studies would be required to clarify whether breast milk miRNAs are biologically functional and contribute to the development of the infant immune system.

The discovery of extracellular miRNAs secreted via microvesicles has triggered an explosion of research activity, 
but has left many unanswered questions about how these molecules are taken up and function in the recipient cell. We demonstrated for the first time that miRNAs secreted by donor cells can be delivered into recipient cells and function as endogenous miRNAs in recipient cells (Zhang et al., 2010). THP-1 cell-derived microvesicles with abundant miR-150 could be delivered into HMEC-1 cells, resulting in the elevation of miR-150 levels in HMEC-1 cells (Zhang et al., 2010). Moreover, we showed that c-Myb, a bona fide target of miR150 , was suppressed by elevated levels of exogenous miR150 , which in turn enhanced cell migration in HMEC-1 cells (Zhang et al., 2010). In addition, increased circulating levels of miR-150 were detected in patients with severe atherosclerosis, and microvesicles isolated from these patients induced a similar response in endothelial cells (Zhang et al., 2010). These results support the idea that miRNA crosstalk between monocytes and endothelial cells may contribute to the development of disease. Similarly, Kosaka et al. detected significant downregulation of a miR-146a reporter when COS7 cells were incubated with a conditioned medium derived from miR-146a-overexpressed cells (Kosaka et al., 2010a). Moreover, they reported that secreted miR-146a, a tumorsuppressive miRNA, suppressed the expression of its target gene ROCK1 in the recipient prostate cancer PC-3M cells, thereby inducing a phenotypic shift to cell growth inhibition (Kosaka et al., 2010a).

Taken together, these studies have demonstrated that microvesicles are not only specifically targeted to recipient cells to exchange proteins, mRNAs, and lipids, but are also able to deliver miRNAs that trigger downstream signaling events. In this pathway, miRNAs are first loaded into small secretory vesicles inside the cells, and then they enter the circulation when the microvesicles are secreted. Using the trafficking system of microvesicles, secreted miRNAs are delivered into recipient cells, where they function as endogenous miRNAs. Considering the relative promiscuity of miRNA species for target mRNA, the impact of microvesicle-mediated transfer of miRNA on the recipient's translational machinery may be quite extensive. The microvesicle pathway may therefore constitute a well-designed mechanism for the local and systemic intercellular transfer of miRNA information, with a complexity superior to that of direct cell-to-cell contacts or secreted soluble factors.

\section{Apoptotic body-mediated miRNA transfer}

Apoptotic bodies are small membranous vesicles that are produced from cells undergoing cell death by apoptosis (Zernecke et al., 2009). Apoptotic endothelial cells at atherosclerotic plaques release apoptotic bodies into the circulation, which were implicated in tissue repair and angiogenesis. Subsequent engulfment of those apoptotic bodies by phagocytes triggers the secretion of cytokines or growth factors (Zernecke et al., 2009). Zernecke et al. showed that endothelial cell-derived apoptotic bodies generated during atherosclerosis contain a panel of miRNAs. The most abundant miRNA of these miRNA species, miR-126, is a key regulator of vascular endothelial growth factor and fibroblast growth factor signaling in endothelial cells (Zernecke et al., 2009). Neighboring vascular cells absorbed these miR-126-containing apoptotic bodies, resulting in the inhibition of the known miR-126 target regulator of $G$ protein signaling 16 (RGS16). Inhibition of RGS16 resulted in the expression of the anti-inflammatory chemokine CXCR4 and increased production of the CXC4 ligand CXCL12 (Zernecke et al., 2009). Because CXCL12 can mediate the recruitment of progenitor cells from the bone marrow to atherosclerotic plaques, thereby limiting plaque size, apoptotic bodies isolated from human patients with atherosclerosis can reduce the size of plaques in different mouse models of atherosclerosis (Zernecke et al., 2009). Thus, this study suggests that dying endothelial cells send alarm signals in the form of apoptotic body-packaged miRNAs to neighboring cells resulting in a healing response that reduces atherosclerosis.

\section{Lipoprotein-mediated miRNA transfer}

A second possibility for extracellular miRNA transfer has been observed when miRNAs are bound to lipoprotein. Recently, Vickers et al. reported that purified fractions of HDL from healthy human plasma contain a number of miRNAs (Vickers et al., 2011). Highly purified HDL that is negative for exosomal marker proteins is rich in small RNA molecules that are 15 to 30 nucleotides in length but devoid of long mRNAs (Vickers et al., 2011). Total RNA extracted from HDL and exosomes isolated from the plasma of healthy individuals revealed that their miRNA profile is distinct (Vickers et al., 2011). A specific miRNA signature of HDL-miRNA complexes was identified in patients with familial hypercholesterolemia, including miR-22, miR-105, and miR-106a (Vickers et al., 2011). The authors further showed that direct delivery of miRNAs to recipient cells can also occur by HDL in a ceramide signaling pathwaydependent manner (Vickers et al., 2011). Thus, native HDL is associated with miRNAs in a way that resembles artificial gene delivery vehicles, acting as a carrier or depot for circulating miRNAs in plasma and facilitating their transport and delivery to recipient cells. Finally, the study by Vickers et al. provides evidence that the miRNAs within HDL alter the cellular miRNA pool and functionally downregulate corresponding miRNA targets (Vickers et al., 2011), suggesting that the miRNA content of HDL is biologically relevant. Collectively, these results indicate that besides its classical role as a delivery vehicle for excess cellular cholesterol, HDL may also function as a transporter of endogenous miRNAs.

\section{RNA-binding protein-mediated miRNA transfer}

Apart from the studies describing miRNA transfer through microvesicles and HDL, other reports have shown that a number of extracellular miRNAs are bound to proteins such 
as AGO2 and NPM1 (Wang et al., 2010; Arroyo et al., 2011; Turchinovich et al., 2011). For example, Wang et al. reported that a significant fraction of extracellular miRNAs is packaged with RNA-binding proteins rather than within shedding vesicles and exosomes (Wang et al., 2010), suggesting a protein-mediated process for miRNA package and export. Indeed, Arroyo et al. observed that the majority of miRNAs in the circulation are present in a non-vesicle-bound AGO2miRNA complex (Arroyo et al., 2011). They speculate that the differences between vesicle-enclosed and AGO2-associated miRNAs may originate from different cell types and reflect cell type-specific miRNA release mechanisms. However, whether there is a protein carrier-based miRNA traffic system remains obscure. There is no direct evidence yet that AGO2- or NPM1-associated miRNAs are actively released from cells, nor is there evidence of their uptake by recipient cells.

\section{Key regulators of miRNA secretion}

Presently, crucial details in our understanding of the mechanism governing the miRNA sorting and secretion are still lacking. Experimental evidence suggests that small RNAs are preferentially encapsulated into microvesicle fractions, although the underlying mechanism remains unknown. For instance, the MC/9 exosomes are enriched in small-size RNAs, but contained few or no ribosomal RNA compared to the donor cells (Valadi et al., 2007). Skog et al. also revealed that microvesicles contain a broad range of small RNAs, including a variety of mRNAs and miRNAs, but lack the ribosomal RNA peaks characteristic of cellular RNA (Skog et al., 2008). Additionally, recent studies indicate that specific miRNA populations are selectively sorted into microvesicles. We demonstrated that blood cells and cultured THP-1 cells actively and selectively package miRNAs into microvesicles and secrete them into the circulation or the culture medium in response to various stimuli (Kosaka et al., 2010a). By profiling RNA isolated from exosomes and their donor cells, Valadi et al. showed that exosomes carry some miRNAs expressed dominantly or at higher levels in exosomes than in the cells (Valadi et al., 2007). Mittelbrunn et al. also showed that certain miRNAs are expressed at higher levels in exosomes than in the parent cells (Mittelbrunn et al., 2011). A recent study demonstrated that the release of exosomal miRNAs is controlled by neutral sphingomyelinase 2 (nSMase2) and uses the ceramide-dependent secretory machinery (Kosaka et al., 2010a). The authors demonstrated that ceramide, whose biosynthesis is tightly controlled by nSMase2, regulates the secretion of exosomal miRNAs. Overexpression of nSMase2 increases extracellular amounts of miRNAs (Kosaka et al., 2010a). By contrast, treatment with a specific siRNA or a chemical compound, GW4869, which can inhibit the enzymatic activity of nSMase2, markedly blocks the secretion of miRNAs as well as exosomes (Kosaka et al., 2010a). Finally, they provided evidence that the endosomal sorting complex required for transport (ESCRT) system is unnecessary for the release of miRNAs (Kosaka et al., 2010a). In agreement with this report, Mittelbrunn et al. also showed that inhibition of exosome production by targeting nSMase 2 with chemical inhibitors or siRNA impairs transfer of miRNAs to antigen-presenting cells (APCs) (Mittelbrunn et al., 2011), further demonstrating a ceramide-triggered, ESCRT-independent machinery for secretion of miRNAs.

How miRNAs are targeted and absorbed by recipient cells also remains unclear. Several groups suggest that this process depends on specific cell surface-targeting motifs on microvesicles and receptors on recipient cells. Microvesicles might be internalized through either direct membrane fusion or endocytosis (Théry et al., 2002; Cocucci et al., 2009; Simons and Raposo, 2009). Therefore, microvesicleenclosed miRNAs may be delivered into recipient cells through receptor-ligand interactions. In addition, HDLmediated miRNA delivery is dependent on a cell surface HDL receptor named scavenger receptor class $B$, type I (SR$\mathrm{BI}$ ), which binds $\mathrm{HDL}$ and mediates the uptake of cholesteryl ester from HDL (Vickers et al., 2011). On the other hand, it is still unknown whether AGO2- or NPM1-associated miRNAs can be absorbed by recipient cells. Nevertheless, additional research is needed to further unravel the mechanisms underlying the uptake of extracellular miRNAs.

\section{FUNCTIONAL ROLES OF SECRETED mIRNAS}

\section{Role of secreted miRNAs in tumor progression}

The tumor microenvironment plays a critical role in cancer progression. Cancer cells can influence normal cells to abandon their homeostatic activities and instead support the neoplastic nature of the tumor. The dynamic crosstalk between cancer cells and normal cells in the microenvironment is crucial to the progression of disease. Crosstalk can occur through secreted molecules and paracrine signaling (Muralidharan-Chari et al., 2010). The secreted molecules are no longer limited to cytokines, chemokines, growth factors or other protein molecules but now also include secreted miRNAs. Skog et al. reported that particular mRNAs and miRNAs are highly enriched in microvesicles from primary human glioblastoma cells (Skog et al., 2008). Glioblastomaderived RNA contained in microvesicles is functional and is taken up by and processed in the human brain microvascular endothelial cell line HBMVEC, generating a functional protein (Skog et al., 2008). These results suggest that the tumorderived microvesicles can modify the surrounding normal cells by changing their translational profile. In addition, the tumor-specific mRNA and miRNAs characteristic of gliomas, such as EGFRvIll and miR-21, could be detected in serum microvesicles of glioblastoma patients (Skog et al., 2008). This finding led to the hypothesis that tumor cells use exosomes to transport genetic information, including miR- 
NAs, to surrounding cells, thereby supporting tumor growth and progression. Furthermore, Ohshima et al. revealed that the let-7 miRNA family was enriched in the extracellular exosomes from a metastatic gastric cancer cell line AZ-P7a, while low metastatic AZ-521, as well as other cancer cell lines, showed no such enrichment (Ohshima et al., 2010). Because let-7 miRNAs generally function as tumor suppressors that target oncogenes such as RAS and high mobility group A2 (HMGA2), they proposed that cancer cells selectively secrete let-7 miRNAs into the extracellular environment via exosomes, reducing the anti-tumorigenic effect within the cells and facilitating oncogenesis and metastasis (Ohshima et al., 2010).

\section{Role of secreted miRNAs in immunological processes}

Secreted miRNAs are also emerging as regulators of immunological processes. Mittelbrunn et al. observed that exosomes from $\mathrm{T}, \mathrm{B}$, and dendritic immune cells contain miRNAs (Mittelbrunn et al., 2011). They demonstrated the existence of antigen-driven unidirectional transfer of miRNAs, such as miR-335, from the T cells to the APCs during T cellAPC cognate immune interactions. This process is mediated by the delivery of $\mathrm{CD63+}$ exosomes during immune synapse formation (Mittelbrunn et al., 2011). Moreover, they showed that miRNAs transferred during immune synapse formation can function in the recipient cell, and suggested that $T$ cellderived miRNAs regulate specific targets in APCs (Mittelbrunn et al., 2011). These results support a mechanism of cellular communication involving antigen-dependent, unidirectional intercellular transfer of miRNAs by exosomes during immune synapse formation.

\section{Role of secreted miRNAs in viral infection}

Epstein-Barr virus (EBV) is a common, potentially oncogenic, $\mathrm{Y}$-herpesvirus and is the first virus known to encode miRNAs (Pfeffer et al., 2004). By characterization of the RNA profile in purified exosomes from EBV-transformed $B$ lymphoblastoid cells (B-LCL), Pegtel et al. showed that the B-LCL exosomes are highly enriched in small RNA species and contain EBVencoded miRNAs (Pegtel et al., 2010). By labeling B-LCL exosomes with fluorescent dye and mimicking exosome transfer from B-LCL to primary immature monocyte-derived dendritic cells (MODCs) using a co-culture model, they demonstrated that EBV-miRNAs are transferred to and accumulate in uninfected MODCs through continuous internalization of exosomes secreted by neighboring B-LCL (Pegtel et al., 2010). These exogenous EBV-miRNAs transferred through exosomes are functional because internalization of exosomes by MODCs leads to a dosedependent, miRNA-mediated repression of CXCL11, a confirmed EBV-miRNA target gene. Finally, they detected EBV-miRNAs in both $B$ cells and uninfected non-B cells in patients with increased EBV load, while EBV-DNA was restricted to the $B$ cell population and was not detectable in T cells or monocytes that make up the bulk of the non-B cell population (Pegtel et al., 2010). The data suggest that EBVmiRNAs are transferred from infected to uninfected cells via exosomes. Furthermore, two recent studies reported that exosomes released from EBV-positive NPC cell lines contain virus-encoded miRNAs. The EBV-miRNAs were detected in the total RNA isolated from HUVEC cells exposed to C666 exosomes with a dose-dependent response (Meckes et al., 2010). Moreover, EBV-miRNAs were detected in the plasma of NPC-xenografted mice and nasopharyngeal carcinoma patients (Gourzones et al., 2010), again demonstrating that extracellular EBV-miRNAs have enough stability and mobility to reach circulating blood.

\section{DIAGNOSTIC AND THERAPEUTIC POTENTIAL OF SECRETED MIRNAS}

Because secreted miRNAs can be detected in biological fluids such as plasma and can reflect the physiological status of the cells and organs they originate from, they could potentially serve as predictive and prognostic biomarkers for diseases. Several studies have suggested using secreted miRNA for diagnostic purposes. For instance, detection of secreted viral miRNAs in the plasma of patients could allow for the detection of infections (Pegtel et al., 2010). Skog et al. reported that the miRNAs characteristic of gliomas could be detected in microvesicles from glioblastoma patient serum (Skog et al., 2008), implying for the first time that miRNAs secreted from tumor cells may provide diagnostic information and aid in therapeutic decisions for cancer patients. Rabinowits et al. isolated exosomes from human plasma and evaluated the levels of total exosome, exosomal small RNA, and specific exosomal miRNAs in controls and lung cancer patients (Rabinowits et al., 2009). They found that the total exosome level and exosomal miRNA concentration were significantly higher in the lung cancer group and that tumor-derived exosomes from the lung cancer patients contained miRNA similar to the corresponding tumor cells (Rabinowits et al., 2009). In another study, Taylor et al. reported that patients diagnosed with ovarian cancer exhibited significantly elevated levels of serum exosomes compared to benign disease or controls and that the levels of circulating exosomes increased as the cancer stage progressed (Taylor and Gercel-Taylor, 2008). Interestingly, miRNA expression profiling in serum exosomes of ovarian cancer patients correlated well with that of their tumors (Taylor and Gercel-Taylor, 2008). Michael et al. showed that exosomes can be readily isolated from the saliva of healthy donors and Sjögren's syndrome patients, and these exosomes contain detectable miRNAs (Gourzones et al., 2010). Because the isolation of exosomes is non-invasive, subsequent characterization of the miRNA expression patterns is straightforward, and the miRNA signatures in secreted 
exosomes parallels the parental tumor cells, exosome profiling holds great promise as an alternate or complementary diagnostic strategy to biopsy profiling and as a screening tool for asymptomatic patients.

Small RNAs including siRNA and miRNA are emerging as promising therapeutic drugs against a wide array of diseases (Ryther et al., 2005; Weiler et al., 2006). Effective delivery of these molecules is crucial to their successful clinical application (Ryther et al., 2005; Weiler et al., 2006). Current techniques for small RNA transfer use viruses or synthetic agents as delivery vehicles. The replacement of these delivery vehicles with a low-toxicity approach is essential for making gene therapy safer. The ability of microvesicles to transfer siRNA and miRNAs raises very exciting possibilities for therapeutic uses. As therapeutic delivery agents, microvesicles would potentially be better tolerated by the immune system because they are natural transporters derived from endogenous cells. Thus, microvesicles derived from cells engineered to express miRNAs or siRNAs may be capable of delivering these small RNAs to local cellular environment. Recently, exosomes have entered the limelight with their ability to transfer exogenous siRNAs into several brain cell types, including neurons, microglia and oligodendrocytes (Alvarez-Erviti et al., 2011). In an excellent study, AlvarezErviti et al. prepared exosomes from dendritic cells that were engineered to express a fusion of the exosomal membrane protein Lamp2b and a rabies virus glycoprotein (RVG) peptide (this peptide derived from RVG can specifically target neuronal cells) (Alvarez-Erviti et al., 2011). By expressing this neuron-targeting protein on the surface of exosomes, filling them with siRNA and injecting them into the bloodstream of mice, the authors achieved specific siRNA delivery to the brain, whereas non-specific uptake of siRNA in other tissues was not observed (Alvarez-Erviti et al., 2011). To show the therapeutic potential of this approach, they delivered siRNA against BACE1, inhibition of which leads to a significant decrease in brain $\beta$-amyloid levels of wild-type mice and is therefore a therapeutic target in Alzheimer's disease (Alvarez-Erviti et al., 2011). Exosome-mediated siRNA delivery resulted in a strong mRNA and protein knockdown of BACE1 in wild-type mice (Alvarez-Erviti et al., 2011). These results point to the potential application of exosomes in small RNA delivery, opening up avenues for therapeutic applications.

\section{CONCLUDING REMARKS AND FUTURE PERSPECTIVES}

The presence of extracellular circulating miRNAs has been detected in a variety of conditions. As observed in many studies, these miRNAs are remarkably stable and are often found inside small membranous vesicles (exosomes, shedding vesicles, and apoptotic bodies) or in association with RNA-binding proteins (HDL, AGO2, and NPM1). Additional findings strongly support the hypothesis that extracellular
miRNAs in these forms are actively secreted from cells, are transported within the extracellular environment and are taken up by recipient cells before their finally binding to target mRNAs, resulting in gene silencing in the recipient cells. Numerous miRNAs can be delivered at once, thus simultaneously regulating multiple target genes and activating a complex network of signaling events in the target cells.

While the biogenesis of extracellular miRNAs has been intensively studied in the recent past, several pertinent issues require further investigation. One central question is to determine how miRNAs are specifically targeted for secretion and recognized for uptake. In particular, what processes mediate the packaging of specific miRNAs into membranous vesicles or with RNA-binding proteins, what triggers miRNA release in different cell types, and which mechanisms are involved in incorporating miRNAs into targeted cells are questions inviting further exploration. Answers to these questions will assist the design of strategies for diagnostic and therapeutic applications.

The exciting research field of miRNA secretion is still in its infancy. Here, we summarize the current views of the secretion and transport of miRNAs and discuss studies that highlight their physiological relevance. We anticipate that future studies in this area will revolutionize the way we perceive how cells communicate, as well as the way we treat various diseases.

\section{ACKNOWLEDGEMENTS}

This work was supported by grants from the National Natural Science Foundation of China (Nos. 90813035, 81101330, 81171661, 30890044, 30772484, 30725008, 30890032, 31071232, 31000323, 90608010, and J1103512), the Natural Science Foundation of Jiangsu Province (No. BK2011013), and the Fundamental Research Funds for the Central Universities (No. 1107020839).

\section{ABBREVIATIONS}

AGO2, Argonaute 2; APC, antigen-presenting cells; B-LCL, EBVtransformed B lymphoblastoid cells; EBV, Epstein-Barr virus; ESCRT, endosomal sorting complex required for transport; $H D L$, high-density lipoprotein; HMGA2, high mobility group A2; miRNA, microRNA; MODC, monocyte-derived dendritic cell; MVB, multivesicular body; NPM1, nucleophosmin 1; nSMase2, sphingomyelinase 2; premiRNA, miRNA precursor; pri-miRNA, primary miRNA transcript; RISC, RNA-induced silencing complex; RGS16, regulator of $G$ protein signaling 16; RNase, ribonuclease; RVG, rabies virus glycoprotein; SR-BI, scavenger receptor class B, type I; 3' UTR, 3' untranslated region

\section{REFERENCES}

Ahmed, K.A., and Xiang, J. (2011). Mechanisms of cellular communication through intercellular protein transfer. J Cell Mol Med 15, 1458-1473.

Alvarez-Erviti, L., Seow, Y., Yin, H., Betts, C., Lakhal, S., and Wood, M.J. (2011). Delivery of siRNA to the mouse brain by systemic 
injection of targeted exosomes. Nat Biotechnol 29, 341-345.

Ambros, V. (2004). The functions of animal microRNAs. Nature 431, 350-355.

Arroyo, J.D., Chevillet, J.R., Kroh, E.M., Ruf, I.K., Pritchard, C.C., Gibson, D.F., Mitchell, P.S., Bennett, C.F., Pogosova-Agadjanyan, E.L., Stirewalt, D.L., et al. (2011). Argonaute2 complexes carry a population of circulating microRNAs independent of vesicles in human plasma. Proc Natl Acad Sci U S A 108, 5003-5008.

Bartel, D.P. (2004). MicroRNAs: genomics, biogenesis, mechanism, and function. Cell 116, 281-297.

Belting, M., and Wittrup, A. (2008). Nanotubes, exosomes, and nucleic acid-binding peptides provide novel mechanisms of intercellular communication in eukaryotic cells: implications in health and disease. J Cell Biol 183, 1187-1191.

Bergsmedh, A., Szeles, A., Henriksson, M., Bratt, A., Folkman, M.J., Spetz, A.L., and Holmgren, L. (2001). Horizontal transfer of oncogenes by uptake of apoptotic bodies. Proc Natl Acad Sci U S A 98, 6407-6411.

Calin, G.A., and Croce, C.M. (2006). MicroRNA signatures in human cancers. Nat Rev Cancer 6, 857-866.

Chen, X., Ba, Y., Ma, L., Cai, X., Yin, Y., Wang, K., Guo, J., Zhang, Y., Chen, J., Guo, X., et al. (2008). Characterization of microRNAs in serum: a novel class of biomarkers for diagnosis of cancer and other diseases. Cell Res 18, 997-1006.

Chen, X., Gao, C., Li, H., Huang, L., Sun, Q., Dong, Y., Tian, C., Gao, S., Dong, H., Guan, D., et al. (2010). Identification and characterization of microRNAs in raw milk during different periods of lactation, commercial fluid, and powdered milk products. Cell Res 20, 1128-1137.

Cocucci, E., Racchetti, G., and Meldolesi, J. (2009). Shedding microvesicles: artefacts no more. Trends Cell Biol 19, 43-51.

Collino, F., Deregibus, M.C., Bruno, S., Sterpone, L., Aghemo, G., Viltono, L., Tetta, C., and Camussi, G. (2010). Microvesicles derived from adult human bone marrow and tissue specific mesenchymal stem cells shuttle selected pattern of miRNAs. PLoS One 5, e11803.

Davis, D.M. (2007). Intercellular transfer of cell-surface proteins is common and can affect many stages of an immune response. Nat Rev Immunol 7, 238-243.

Deregibus, M.C., Cantaluppi, V., Calogero, R., Lo lacono, M., Tetta, C., Biancone, L., Bruno, S., Bussolati, B., and Camussi, G. (2007). Endothelial progenitor cell derived microvesicles activate an angiogenic program in endothelial cells by a horizontal transfer of mRNA. Blood 110, 2440-2448.

Dunning Hotopp, J.C. (2011). Horizontal gene transfer between bacteria and animals. Trends Genet 27, 157-163.

Esquela-Kerscher, A., and Slack, F.J. (2006). Oncomirs - microRNAs with a role in cancer. Nat Rev Cancer 6, 259-269.

Gourzones, C., Gelin, A., Bombik, I., Klibi, J., Vérillaud, B., Guigay, J., Lang, P., Témam, S., Schneider, V., Amiel, C., et al. (2010). Extracellular release and blood diffusion of BART viral micro-RNAs produced by EBV-infected nasopharyngeal carcinoma cells. Virol J 7, 271.

Hanke, M., Hoefig, K., Merz, H., Feller, A.C., Kausch, I., Jocham, D., Warnecke, J.M., and Sczakiel, G. (2010). A robust methodology to study urine microRNA as tumor marker: microRNA-126 and microRNA-182 are related to urinary bladder cancer. Urol Oncol 28, 655-661.
Hata, T., Murakami, K., Nakatani, H., Yamamoto, Y., Matsuda, T., and Aoki, N. (2010). Isolation of bovine milk-derived microvesicles carrying mRNAs and microRNAs. Biochem Biophys Res Commun 396, 528-533.

He, L., and Hannon, G.J. (2004). MicroRNAs: small RNAs with a big role in gene regulation. Nat Rev Genet 5, 522-531.

Holmgren, L. (2010). Horizontal gene transfer: you are what you eat. Biochem Biophys Res Commun 396, 147-151.

Holmgren, L., Szeles, A., Rajnavölgyi, E., Folkman, J., Klein, G., Ernberg, I., and Falk, K.I. (1999). Horizontal transfer of DNA by the uptake of apoptotic bodies. Blood 93, 3956-3963.

Hunter, M.P., Ismail, N., Zhang, X.L., Aguda, B.D., Lee, E.J., Yu, L.B., Xiao, T., Schafer, J., Lee, M.L.T., Schmittgen, T.D., et al. (2008). Detection of microRNA expression in human peripheral blood microvesicles. PLoS One 3, e3694.

Hutvágner, G., McLachlan, J., Pasquinelli, A.E., Bálint, E., Tuschl, T., and Zamore, P.D. (2001). A cellular function for the RNAinterference enzyme Dicer in the maturation of the let-7 small temporal RNA. Science 293, 834-838.

Ji, X., Takahashi, R., Hiura, Y., Hirokawa, G., Fukushima, Y., and Iwai, N. (2009). Plasma miR-208 as a biomarker of myocardial injury. Clin Chem 55, 1944-1949.

Keeling, P.J., and Palmer, J.D. (2008). Horizontal gene transfer in eukaryotic evolution. Nat Rev Genet 9, 605-618.

Khvorova, A., Reynolds, A., and Jayasena, S.D. (2003). Functional siRNAs and miRNAs exhibit strand bias. Cell 115, 209-216.

Kosaka, N., Iguchi, H., Yoshioka, Y., Takeshita, F., Matsuki, Y., and Ochiya, T. (2010a). Secretory mechanisms and intercellular transfer of microRNAs in living cells. J Biol Chem 285, 17442-17452.

Kosaka, N., Izumi, H., Sekine, K., and Ochiya, T. (2010b). microRNA as a new immune-regulatory agent in breast milk. Silence 1, 7

Laterza, O.F., Lim, L., Garrett-Engele, P.W., Vlasakova, K., Muniappa, N., Tanaka, W.K., Johnson, J.M., Sina, J.F., Fare, T.L., Sistare, F. D., et al. (2009). Plasma MicroRNAs as sensitive and specific biomarkers of tissue injury. Clin Chem 55, 1977-1983.

Lawrie, C.H., Gal, S., Dunlop, H.M., Pushkaran, B., Liggins, A.P., Pulford, K., Banham, A.H., Pezzella, F., Boultwood, J., Wainscoat, J.S., et al. (2008). Detection of elevated levels of tumourassociated microRNAs in serum of patients with diffuse large Bcell lymphoma. Br J Haematol 141, 672-675.

Lee, Y., Ahn, C., Han, J., Choi, H., Kim, J., Yim, J., Lee, J., Provost, P., Rådmark, O., Kim, S., et al. (2003). The nuclear RNase III Drosha initiates microRNA processing. Nature 425, 415 419.

Lee, Y., Kim, M., Han, J., Yeom, K.H., Lee, S., Baek, S.H., and Kim, V. N. (2004). MicroRNA genes are transcribed by RNA polymerase II. EMBO J 23, 4051-4060.

Lund, E., Güttinger, S., Calado, A., Dahlberg, J.E., and Kutay, U. (2004). Nuclear export of microRNA precursors. Science 303, 95-98.

Luo, S.S., Ishibashi, O., Ishikawa, G., Ishikawa, T., Katayama, A., Mishima, T., Takizawa, T., Shigihara, T., Goto, T., Izumi, A., et al. (2009). Human villous trophoblasts express and secrete placentaspecific microRNAs into maternal circulation via exosomes. Biol Reprod 81, 717-729.

Meckes, D.G. Jr, Shair, K.H.Y., Marquitz, A.R., Kung, C.P., Edwards, R.H., and Raab-Traub, N. (2010). Human tumor virus utilizes exosomes for intercellular communication. Proc Natl Acad Sci U S 
A 107, 20370-20375.

Mitchell, P.S., Parkin, R.K., Kroh, E.M., Fritz, B.R., Wyman, S.K., Pogosova-Agadjanyan, E.L., Peterson, A., Noteboom, J., O'Briant, K.C., Allen, A., et al. (2008). Circulating microRNAs as stable blood-based markers for cancer detection. Proc Natl Acad Sci U S A 105, 10513-10518.

Mittelbrunn, M., Gutiérrez-Vázquez, C., Villarroya-Beltri, C., González, S., Sánchez-Cabo, F., González, M.A., Bernad, A., and Sánchez-Madrid, F. (2011). Unidirectional transfer of microRNAloaded exosomes from $\mathrm{T}$ cells to antigen-presenting cells. Nat Commun 2, 282.

Müller, G., Schneider, M., Biemer-Daub, G., and Wied, S. (2011). Microvesicles released from rat adipocytes and harboring glycosylphosphatidylinositol-anchored proteins transfer RNA stimulating lipid synthesis. Cell Signal 23, 1207-1223.

Muralidharan-Chari, V., Clancy, J.W., Sedgwick, A., and D'SouzaSchorey, C. (2010). Microvesicles: mediators of extracellular communication during cancer progression. J Cell Sci 123, 1603-1611.

Ogawa, R., Tanaka, C., Sato, M., Nagasaki, H., Sugimura, K., Okumura, K., Nakagawa, Y., and Aoki, N. (2010). Adipocytederived microvesicles contain RNA that is transported into macrophages and might be secreted into blood circulation. Biochem Biophys Res Commun 398, 723-729.

Ohshima, K., Inoue, K., Fujiwara, A., Hatakeyama, K., Kanto, K., Watanabe, Y., Muramatsu, K., Fukuda, Y., Ogura, S., Yamaguchi, K., et al. (2010). Let-7 microRNA family is selectively secreted into the extracellular environment via exosomes in a metastatic gastric cancer cell line. PLoS One 5, e13247.

Park, N.J., Zhou, H., Elashoff, D., Henson, B.S., Kastratovic, D.A., Abemayor, E., and Wong, D.T. (2009). Salivary microRNA: discovery, characterization, and clinical utility for oral cancer detection. Clin Cancer Res 15, 5473-5477.

Pegtel, D.M., Cosmopoulos, K., Thorley-Lawson, D.A., van Eijndhoven, M.A.J., Hopmans, E.S., Lindenberg, J.L., de Gruijl, T.D., Würdinger, T., and Middeldorp, J.M. (2010). Functional delivery of viral miRNAs via exosomes. Proc Natl Acad Sci U S A 107, 6328-6333.

Pfeffer, S., Zavolan, M., Grässer, F.A., Chien, M.C., Russo, J.J., Ju, J. Y., John, B., Enright, A.J., Marks, D., Sander, C., et al. (2004). Identification of virus-encoded microRNAs. Science 304, 734-736.

Rabinowits, G., Gerçel-Taylor, C., Day, J.M., Taylor, D.D., and Kloecker, G.H. (2009). Exosomal microRNA: a diagnostic marker for lung cancer. Clin Lung Cancer 10, 42-46.

Ratajczak, J., Miekus, K., Kucia, M., Zhang, J., Reca, R., Dvorak, P., and Ratajczak, M.Z. (2006). Embryonic stem cell-derived microvesicles reprogram hematopoietic progenitors: evidence for horizontal transfer of mRNA and protein delivery. Leukemia 20 , 847-856.

Rechavi, O., Goldstein, I., and Kloog, Y. (2009). Intercellular exchange of proteins: the immune cell habit of sharing. FEBS Lett 583, 1792-1799.

Ryther, R.C., Flynt, A.S., Phillips, J.A. 3rd, and Patton, J.G. (2005).
siRNA therapeutics: big potential from small RNAs. Gene Ther 12 , 5-11.

Schwarz, D.S., Hutvágner, G., Du, T., Xu, Z., Aronin, N., and Zamore, P.D. (2003). Asymmetry in the assembly of the RNAi enzyme complex. Cell 115, 199-208.

Simons, M., and Raposo, G. (2009). Exosomes-vesicular carriers for intercellular communication. Curr Opin Cell Biol 21, 575-581.

Skog, J., Würdinger, T., van Rijn, S., Meijer, D.H., Gainche, L., SenaEsteves, M., Curry, W.T. Jr, Carter, B.S., Krichevsky, A.M., and Breakefield, X.O. (2008). Glioblastoma microvesicles transport RNA and proteins that promote tumour growth and provide diagnostic biomarkers. Nat Cell Biol 10, 1470-1476.

Taylor, D.D., and Gercel-Taylor, C. (2008). MicroRNA signatures of tumor-derived exosomes as diagnostic biomarkers of ovarian cancer. Gynecol Oncol 110, 13-21.

Théry, C., Zitvogel, L., and Amigorena, S. (2002). Exosomes: composition, biogenesis and function. Nat Rev Immunol 2, 569-579.

Turchinovich, A., Weiz, L., Langheinz, A., and Burwinkel, B. (2011). Characterization of extracellular circulating microRNA. Nucleic Acids Res 39, 7223-7233.

Valadi, H., Ekström, K., Bossios, A., Sjöstrand, M., Lee, J.J., and Lötvall, J.O. (2007). Exosome-mediated transfer of mRNAs and microRNAs is a novel mechanism of genetic exchange between cells. Nat Cell Biol 9, 654-659.

van Rooij, E., and Olson, E.N. (2007). MicroRNAs: powerful new regulators of heart disease and provocative therapeutic targets. $J$ Clin Invest 117, 2369-2376.

Vickers, K.C., Palmisano, B.T., Shoucri, B.M., Shamburek, R.D., and Remaley, A.T. (2011). MicroRNAs are transported in plasma and delivered to recipient cells by high-density lipoproteins. Nat Cell Biol 13, 423-433.

Wang, K., Zhang, S., Marzolf, B., Troisch, P., Brightman, A., Hu, Z., Hood, L.E., and Galas, D.J. (2009). Circulating microRNAs, potential biomarkers for drug-induced liver injury. Proc Natl Acad Sci U S A 106, 4402-4407.

Wang, K., Zhang, S.L., Weber, J., Baxter, D., and Galas, D.J. (2010). Export of microRNAs and microRNA-protective protein by mammalian cells. Nucleic Acids Res 38, 7248-7259.

Weiler, J., Hunziker, J., and Hall, J. (2006). Anti-miRNA oligonucleotides (AMOs): ammunition to target miRNAs implicated in human disease? Gene Ther 13, 496-502.

Yuan, A., Farber, E.L., Rapoport, A.L., Tejada, D., Deniskin, R., Akhmedov, N.B., and Farber, D.B. (2009). Transfer of microRNAs by embryonic stem cell microvesicles. PLoS One 4, e4722.

Zernecke, A., Bidzhekov, K., Noels, H., Shagdarsuren, E., Gan, L., Denecke, B., Hristov, M., Köppel, T., Jahantigh, M.N., Lutgens, E. et al. (2009). Delivery of microRNA-126 by apoptotic bodies induces CXCL12-dependent vascular protection. Sci Signal 2, ra81.

Zhang, Y.J., Liu, D.Q., Chen, X., Li, J., Li, L.M., Bian, Z., Sun, F., Lu, J. W., Yin, Y.A., Cai, X., et al. (2010). Secreted monocytic miR-150 enhances targeted endothelial cell migration. Mol Cell 39, 133-144. 